\title{
Optical and Morphological Studies of Ga Doped ZnO Nanocrystals
}

\author{
Achamma George, Shajeem J \\ Department of Physics, Catholicate College, Pathanamthitta, Kerala India pin: 689645 \\ Email:gachamma@gmail.com
}

\begin{abstract}
ZnO}$ undoped and doped nanocrystals with spherical morphology have been synthesized by à cost effective hydrolysis technique. X-ray diffraction analysis was carried out to evaluate the contribution of dopant ion lattice parameters. The crystallite size calculated is in good agreement with the TEM results. For pristine samples, PL is broad in nature with maxima at various positions in visible region of electromagnetic spectrum. The emission spectra of most $\mathrm{ZnO}$ sample show a near-band-edge line $(\lambda=366 \mathrm{~nm})$ followed by a deep level luminescence in lower energy regime $(\lambda=453 \mathrm{~nm}, 546 \mathrm{~nm}$ and $634 \mathrm{~nm})$. In the present case, the higher energy band $(366 \mathrm{~nm})$ is assigned to the occurrence of free excitons' recombination through an exciton-exciton collision process. The Ga doped luminescence spectra, the emission peaks are at $364 \mathrm{~nm}, 442 \mathrm{~nm} 544$ and $635 \mathrm{~nm} . \mathrm{NBE}$ emission had a decrement in the intensity along with shift towards lower wavelength and the 442 emission had a increase in intensity also the emission due to other intrinsic defects were depressed with Ga doping. This may be attributed to the decrease in particle size of doped samples.
\end{abstract}

Keywords: nanocrystals, luminescence, excitons, $\mathrm{ZnO}$

\section{Introduction}

Nano sized Zinc oxide $(\mathrm{ZnO})$ doped with different ions have improved its applicability, as a material for blue/UV optoelectronics, as an alternative to $\mathrm{GaN}$, as a cheap material for electronic circuits which are conductive, transparent in the visible region and as semiconductor spintronics [1]. ZnO crystallizes preferentially in the hexagonal wurtzite-type structure with common name 'zincite'. Earlier, we have also reported cerium doped $\mathrm{ZnO}$ with its detailed $\mathrm{X}$ ray diffraction studies[2] In order to extend the domain of our research on $\mathrm{ZnO}$ and to meet the industrial needs of low-cost, large-scale production and to systematically study the formation theory of $\mathrm{ZnO}$ materials, controlled synthesis under mild conditions is very crucial. The synthesis of $\mathrm{ZnO}$ nanocrystals via low temperature refluxing technique is done. Post transition element gallium $(\mathrm{Ga})$ is less reactive and more resistive to oxidation. The covalent bond lengths of $\mathrm{Ga}-\mathrm{O}$ are slightly smaller than that of $\mathrm{Zn}-\mathrm{O}$, which will make the deformation of the $\mathrm{ZnO}$ lattice small even in the case of high $\mathrm{Ga}$ concentration [3]. Different authors have also reported the synthesis of $\mathrm{Ga}$ doped $\mathrm{ZnO}[4, \mathbf{5}, \mathbf{6 , 7 ]}$. In all these reports, the synthesis was performed via time consuming, expensive procedures. Herein, we have adopted the refluxing technique to synthesize $\mathrm{ZnO}: \mathrm{Ga}$. In this procedure, the synthesis temperature and the processing time is appreciably low than the other traditional synthesis routes. The preparation of $\mathrm{Ga}$ doped $\mathrm{ZnO}$ via this route has not been reported so far, to the best of authors' knowledge. In the present work, we have answered issues concerning the effect of $\mathrm{Ga}$ ion concentration on morphostructural parameters of $\mathrm{ZnO}$. The works pursued include synthesis of $\mathrm{ZnO}$ nanocrystals, X-ray diffraction analysis, morphology, size determination and studies on radiative recombination processes for pristine and $\mathrm{Ga}$ doped $\mathrm{ZnO}$ nanopowders.

\subsection{Synthesis:}

\section{Experimental}

The GR grade (purity> 99\%) Zinc acetate $\left(\mathrm{Zn}\left(\mathrm{CH}_{3} \mathrm{COO}\right)_{2} \cdot 2 \mathrm{H}_{2} \mathrm{O}\right)$, gallium nitrate $\left(\mathrm{Ga}\left(\mathrm{NO}_{3}\right)_{3} \cdot 9 \mathrm{H}_{2} \mathrm{O}\right)$, urea $\left(\mathrm{CH}_{2} \mathrm{NH}_{2} \mathrm{COOH}\right)$, and ethylene glycol $\left(\mathrm{C}_{2} \mathrm{H}_{6} \mathrm{O}_{2}\right)$, were procured from E. Merck India. No further purification of as-obtained precursor was carried out. In a typical experiment, $0.5 \mathrm{~g}$ of zinc acetate was dissolved in $50 \mathrm{ml}$ of ethylene glycol. Around $1.5 \mathrm{~g}$ of urea was added into this solution and the temperature is raised to $150^{\circ} \mathrm{C}$ and maintained at this value for $2 \mathrm{hr}$. A white precipitate of $\mathrm{ZnO}$ was obtained which was finally separated by centrifugation and washed several times with quadruple distilled water and methanol to remove traces impurities and thereafter dried in room conditions. For $\mathrm{Ga}(4 \mathrm{~mol} \%)$ doped $\mathrm{ZnO}$ nanocrystals, same procedure was followed except that, appropriate amount of gallium nitrate was dissolved with the solution at the beginning of the reaction process. Both the undoped and doped powders were calcined at $500^{\circ} \mathrm{C}$. 


\subsection{Characterization:}

The phase identification of as-synthesized $\mathrm{ZnO}$ and $\mathrm{Ga}$-doped $\mathrm{ZnO}$ nanocrystals was performed on Bruker AXS D8 Advance instrument with $\mathrm{Cu}-\mathrm{K} \alpha$ target $\left(\lambda=1.540604 \mathrm{~A}^{\circ}\right)$ at room temperature. The X-ray generator was operated at $30 \mathrm{kV}$ with a scan speed of 2 degree/min. TEM measurements were carried out on Jeol/JEM 2100 with a high resolution of $2.4 \mathrm{~A}^{\circ}$. Normalized photoluminescence spectra have been measured using Horiba Fluorolog Fluorescence spectrophotometer. WITEC ALPHA300 RA was used for Atomic Force Microscopy (AFM)

\subsection{XRD RESULTS:}

\section{Results}

To ascertain the crystalline nature of pristine and doped $\mathrm{ZnO}$ nanocrystalline powders, $\mathrm{X}$-Ray diffraction studies were performed. The patterns for the same is shown in (Fig. 1) along with standard pattern from Joint committee powder diffraction standards (JCPDS) file No 36-1451. In both cases, no additional signatures were observed. It is anticipated that the $\mathrm{Ga}^{3+}$ has substituted the $\mathrm{Zn}^{2+}$ sites due to their matching radii. As the ionic radius of $\mathrm{Ga}^{3+}(0.062 \mathrm{~nm})$ are smaller than $\mathrm{Zn}^{2+}(0.074 \mathrm{~nm})$, so the substitutional replacement could be easily possible. The distortion in $\mathrm{Ga}$ doped $\mathrm{ZnO}$ (GZO) crystal structure is less than other element like $\mathrm{Al}$ doped $\mathrm{ZnO}$ due to the lower approximate ionic radii of $\mathrm{Ga}^{3+}$ to $\mathrm{Zn}^{2+}[4]$. The Madelung energy and solubility limit can possibly affect the substitution of $\mathrm{Zn}$ atoms by dopants. Excessive doping atoms occupy the interstitial positions or form gallium oxides (more than 5 at.\%), which entirely distort $\mathrm{ZnO}$ crystal structure. Interstitial $\mathrm{Ga}$ are surrounded by three oxygen atoms and have a weak bond with oxygen atoms, which boost the Madelung energy of the $\mathrm{ZnO}$ crystal structure. The peak broadening features in the present case has been assigned to the smaller crystallite size (also confirmed from TEM results). The variation of unit cell parameters with dopant concentration is given in the (Table 1). However, GZO has the drawback of low durability in humid environment. Furthermore, expensiveness of Ga compounds is another drawback in GZO especially for mass production [4]. The volume increment of the lattice with Ga doping indicates the presence of some type of intrinsic defects of vacancies, point defects, dislocation etc.

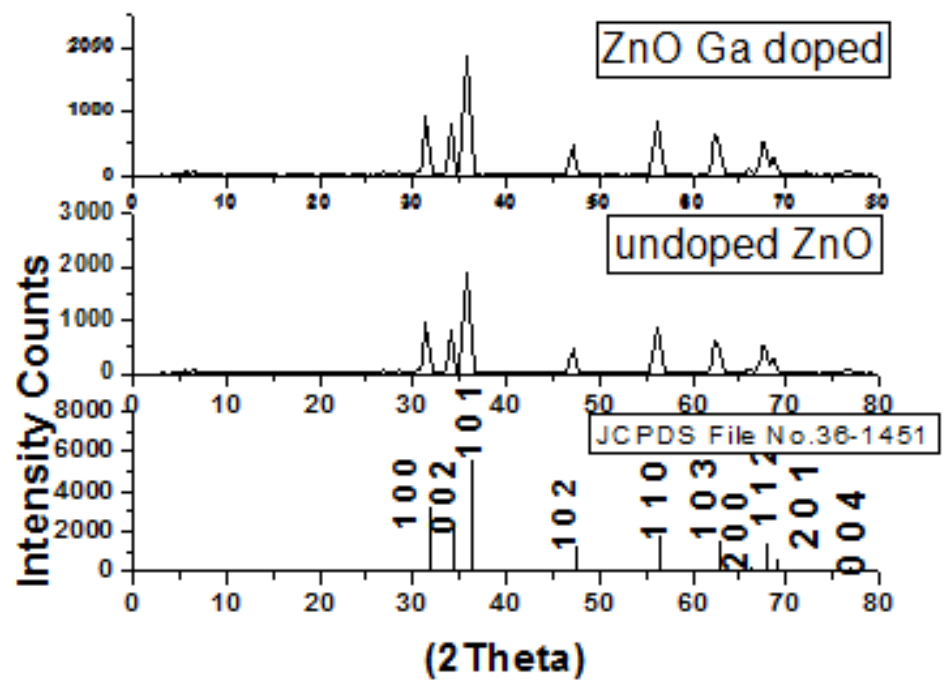

Figure $1 \mathrm{XRD}$ patterns of undoped $\mathrm{ZnO} \& 4 \mathrm{~mol} \%$ doped $\mathrm{Ga}$ in $\mathrm{ZnO}$.

At lower concentration of $\mathrm{Ga}^{3+}$ the defects get neutralized and do not influence crystallite size, but as the Ga concentration increases, the size becomes thickness dependent which effect the density and increases the volume which is further supported by change in axial ratio. The apparent crystallite size is calculated using Scherrer equation and is approximated at $21 \mathrm{~nm}$ for undoped and $19 \mathrm{~nm}$ for the doped $\mathrm{ZnO}$. The equation for which is

$d=\frac{k \lambda}{\beta \cos \theta}$

Where $k$ is close to unity, $\beta$ is FWHM, $\theta$ is Bragg angle of $\{\mathrm{h} \mathrm{kl}\}$ reflections, and $d$ is the crystallite size and $\lambda$ is wavelength of X-rays. The reduction of $d$ crystallite size value of due to Ga doping can be attributed due to the replacement of $\mathrm{Zn}^{2+}$ ions by $\mathrm{Ga}^{3+}$ ions as the radius of $\mathrm{Zn}^{2+}$ is greater than that of $\mathrm{Ga}^{3+}$ ions. 
Table 1 Lattice parameters of Ga doped and undoped $\mathrm{ZnO}$.

\begin{tabular}{|c|c|c|c|c|}
\hline Conc. & a & c & c/a & volume \\
\hline Undoped & 3.27 & 5.07 & 1.55 & 47.14 \\
\hline $4 \mathrm{~mol} \%$ & 3.27 & 5.26 & 1.60 & 48.88 \\
\hline
\end{tabular}

Figure 2 TEM images of undoped, Ga doped $\mathrm{ZnO}$ and EDX spectra

\subsection{Morphology \& TEM Results:}

The TEM pattern for pristine $\mathrm{ZnO}$ nanocrystals is shown in (Fig.2). The crystallites have spherical morphology. There are numerous factors that can affect the shape and size of the crystallites during synthesis procedure. In the present case, the spherical morphology is attributed to the crucial role played by the ethylene glycol during synthesis of $\mathrm{ZnO}$ nanocrystals. As an important non-ionic surfactant with uniform and ordered chain like structure, ethylene glycol is mainly absorbed at the surface of the metal oxides. It is known that the organic ligands with high binding affinities can promote self-assembly driven by the interaction between the surface absorbed ligands instead of between the particles themselves. In addition, the ligands involved self assembly is likely to generate single crystalline architecture that usually couldn't be obtained from the conventional processes. Hence, we anticipate that during the self assembly, at low concentrations, $\mathrm{ZnO}$ particles rotated themselves and aggregated through ethylene glycol while sharing same crystallographic planes leading to spherical morphology.

To confirm the size of the $\mathrm{ZnO}$ powders, size calculations were performed using TEM experiment. The TEM pattern for undoped is shown in (Fig. 2) and 4mol\% Ga doped is shown in (Fig. 2). As noticed, an average crystallite size of $21.93 \mathrm{~nm}$ of undoped and around $20 \mathrm{~nm}$ of $\mathrm{Ga}$ doped is approximated in the present case. A good correlation is found to exist between mathematical calculations from XRD analysis and those obtained via TEM studies. The decrease in grain size and uniformity of the Ga doped $\mathrm{ZnO}$ grains has been already reported by R Ebrahimifard et.al[3].Energy-dispersive X-ray Spectroscopy(EDX) (Fig. 2) also shows the presence of Ga.

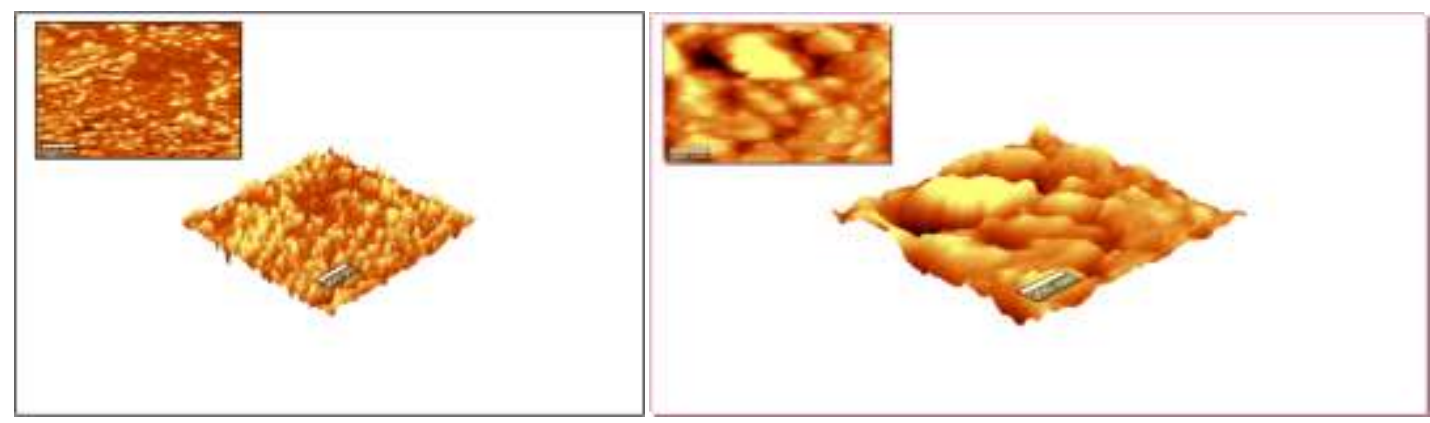

Figure 3 AFM images(inset 2D view) of doped and undoped

The 3D view of the $\mathrm{ZnO}$ nanocrystals on a glass substrate over a scanning area of $3.63 \mu \mathrm{m}^{2}$ and that of Ga doped $\mathrm{ZnO}$ nanocrystals over a scanning area $3.10 \mu \mathrm{m}^{2}$ are shown in the Fig.3. The inset 2D view, reveals the aggregation of the crystallites to form large grains. The peak to peak roughness of undoped was 59nm whereas that of doped was found to be $110 \mathrm{~nm}$.

\subsection{Photoluminescence Results:}

Luminescence results shows that the near-band-edge(NBE) luminescence which is assigned to donorto-free-hole recombination. The intensity of the near-band-edge transition is decreased markedly by the doping. Doping yielded a band-gap widening $(\Delta \lambda=2 \mathrm{~nm})$. The sizable amounts of Stokes shift and PL broadening can be due to effect of photo created carriers.[3] The emission spectrum of pristine $\mathrm{ZnO}$ contains various 4 maxima at $330 \mathrm{~nm}$ excitation wavelength (Fig. 3). The bands are present in complete visible regions and are attributed to various types of defects present in the as-synthesized $\mathrm{ZnO}$ role. In luminescence process vacancies and interstitials play a key role. There are seven defect states which were observed by Xu et. al.[8] by using local 
density approximation in density functional theory for $\mathrm{ZnO}$ system. The probable defects, here can be interstitial $\mathrm{Zn}$ or $\mathrm{O}\left(\mathrm{Zn}_{\mathrm{i}}\right.$ or $\left.\mathrm{O}_{\mathrm{i}}\right)$, zinc vacancy $\left(\mathrm{V}_{\mathrm{zn}}\right)$ or the oxygen antisite $\left(\mathrm{O}_{\mathrm{Zn}}\right)$. The emission spectra of most $\mathrm{ZnO}$ sample show a near-band-edge line $(\lambda=366 \mathrm{~nm})$ followed by a deep level luminescence in lower energy regime $(\lambda=453 \mathrm{~nm}, 546 \mathrm{~nm}$, and $634 \mathrm{~nm})$. Higher wavelength band originates from the various defects depending upon the growth technique and can decrease the emission efficiency of the UV emission while increasing the lasing action threshold. Hence, the optical quality is evaluated using intensity ratios between near UV and the green bands. Luminescence features also depend on other crucial factors which includes solvent, atmosphere, starting materials, etc and is attributed to different processing conditions (quenching centers) during synthesis process. In the present case, the higher energy band $(366 \mathrm{~nm})$ is assigned to the occurrence of free excitons recombination through an exciton-exciton collision process. On the other hand, the lower energy aqua-blue (453nm) emission band is assigned to the presence of oxygen vacancies or to some type of new defects formed during synthesis of $\mathrm{ZnO}$. The yellow band at $546 \mathrm{~nm}$ is thought to have evolved due to transition from shallow donor to a deep acceptor above the top of the valence band and is tentatively assigned to acceptor involved in Vzn or V-zn shallow donor complexes [9]. The red band at $634 \mathrm{~nm}$ has been assigned to other type of intermediate trapping state. To exactly claim about the exact nature of defects, further experimentation like calcining effects (on PL) of $\mathrm{ZnO}$ in oxygen or ammonia atmosphere or ESR mapping is required and thus, paves the way for further research. The Ga doped luminescence spectra, shown in (Fig. 4) the emission peaks are at a near-band-edge line $(\lambda=364 \mathrm{~nm})$ followed by a deep level luminescence in lower energy regime $(\lambda=442 \mathrm{~nm}, 544 \mathrm{~nm}$, and $635 \mathrm{~nm})$ similar to the undoped $\mathrm{ZnO}$.NBE emission wavelength is decreased or shifts towards high energy region so blue shift [3] may be along with decrement of intensity of NBE emission[7]. There is change in NBE emission by Ga doping as reported in Park et al.[10] which may be accounted due to particle size decrement. However there is a broad increase in the intensity of peak at 440nm.It may be due to oxygen vacancy defects formed during synthesis of $\mathrm{Ga}$ doped $\mathrm{ZnO}$. The emissions $544 \mathrm{~nm}$ and $635 \mathrm{~nm}$ may be due to similar defects of undoped $\mathrm{ZnO}$.

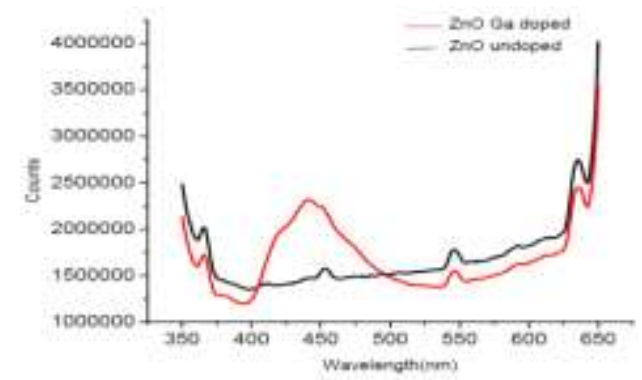

Figure 4 PL spectra of Undoped and Ga doped $\mathrm{ZnO}$.

\section{Conclusion}

$\mathrm{ZnO}$ nanocrystals with spherical morphology have been synthesized by a cost effective hydrolysis technique.XRD studies showed that undoped and doped nanocrystals are phase pure indicating that $\mathrm{Ga}^{3+}$ has substituted the $\mathrm{Zn}^{2+}$ sites due to their matching radii. Spherical morphology is designated to the crucial role played by the ethylene glycol during synthesis. The crystallite size calculated by Scherrer equation value is in good agreement with the TEM results for Ga doped nanocrystals is around 20nm.A decrease in grain size compared to the undoped nanocrystals was observed. For pristine samples, PL had several maxima at various positions in visible region of electromagnetic spectrum. For $\mathrm{Ga}$ doped nanocrystals, the emission intensity increased at 440nm emission and the other defect emission of PL from oxygen or zinc defects were as it is. The NBE emission had a blue shift towards lower wavelength along with intensity decrement in Ga doped samples which may due to the decrease in particle size of doped samples.

\section{Acknowledgements}

The author owes sincere thanks to facilities given STIC Kochi University for XRD and TEM, SAIF DST MG university for AFM and PL measurement facilities.

\section{References}

[1]. C. Klingshirn, ZnO : Materials ,Physics and Application Phys. Stat. Sol. (b), 244 (2007) pp. 3027-3073.

[2]. Achamma George, S K. Sharma, Santa Chawla, M.M. Malik, M.S. Qureshi Detailed of X-ray diffraction and photoluminescence studies of Ce doped ZnO Nanocrystals Journal of Alloys and Compounds 509 (2011) 5942-5946

[3]. T. Makino, Y. Segawa, S. Yoshida, A. Tsukazaki, A. Ohtomo, and M. Kawasaki Gallium concentration dependence of roomtemperature near-band-edge luminescence in n -type ZnO : Ga Applied Physics Letters 85, 759 (2004)

[4]. Reza Ebrahimifard, Mohammad Reza Golobostanfard, Hossein Abdizadeh, Sol-gel derived Al and Ga co-doped ZnO thin films:An optoelectronic study Applied Surface Science 290 (2014) 252- 259.

[5]. Growth Of Epitaxial P-Type Zno Thin Films By Codoping Of Ga and N Manoj Kumar, Tae-Hwan Kim, Sang-Sub Kim, and Byung-Teak Leea_Applied Physics Letters 89, 1121032006 
[6]. S. Chu, J. H. Lim, L. J. Mandalapu, Z. Yang, L. Li, and J. L. Liua Sb-doped p-ZnO/Ga-doped n-ZnO homo junction ultraviolet light emitting diodes Applied Physics Letters 92, 1521032008

[7]. Z.F. Liu,1,4 F.K. Shan,1 J.Y. Sohn,2 S.C. Kim,2 G.Y. Kim,3 Y.X. Li,4 \& Y.S. Yu1,Photoluminescence of ZnO:Ga Thin Films Fabricated by Pulsed Laser Deposition Technique Journal of Electroceramics, 13, 183-187, 2004

[8]. P.S. Xu, Y.M. Sun, C.S. Shi, F.Q. Xu, H.B. Pan, The electronic structure and spectral properties $\mathrm{P}$ of $\mathrm{ZnO}$ and its defects Nucl. Instrum. Methods Phys. Res. B 199 (2003) 286-290.

[9]. M.A. Reshchikov, H. Morkoc, B. Nemeth, J. Nause, J. Xie, B. Hertog, A. Osinsky, Luminescence properties of defects in $\mathrm{ZnO}$ Physics B 401-402 (2007) 358-361.

[10]. H. C. Park and D. Byun B. Angadi, D. Hee Park, and W. K. ChoiJ. W. Choi Y. S. Jung Photoluminescence of Ga-doped ZnO film grown on c- $\mathrm{Al}_{2} \mathrm{O} 3$ (0001) by plasma-assisted molecular beam epitaxy Journal Of Applied Physics 102, 073114 